\title{
Effective Collaborative Communication in Hospice Care
}

\author{
Ami Bhatt* and Anne Mitchell \\ Oakland University, 2200 N Squirrel Rd, Rochester, MI 48309, United States
}

Received: November 22, 2014; Accepted: February 05, 2015; Published: February 21, 2015

*Corresponding author: Ami Bhatt, DNP, MBA, CHPN, CHCI, RN, Oakland University, 2200 N Squirrel Rd, Rochester, MI 48309, United States, E-mail: abhatt22@msn.com

\begin{abstract}
Interdisciplinary collaboration and teamwork are necessary components for collaborative communication to occur between team members in a hospice admissions setting. Team interactions require trust, confidence and an equal effort by all team members. Effective communication can be achieved through collaborative communication between team members in Interdisciplinary Team (IDT) meetings and research has been done to support this concept. However, there is a dearth of research regarding overall collaborative communication in teams and specifically data examining communication in admission teams between clinical and non-clinical team members is limited. The study tested a survey instrument that will be piloted for future research efforts to evaluate the collaborative communication between the clinical and nonclinical team members in the hospice admissions setting. Interdisciplinary collaboration in a hospice and palliative care setting is influenced by effective communication.
\end{abstract}

Keywords: Communication; collaboration; Team work; Interdisciplinary; Teams; palliative; Effective communication; Hospice

\section{Introduction}

Communication can be both verbal and nonverbal with subtypes that can include positive and negative communication, open and closed communication as well as effective and ineffective communication. Communication is defined as "a process by which information is exchanged between individuals through a ommon system of symbols, signs or behavior" (http://www.merriam-webster.com/dictionary/communication). Communication has to be beneficial to all members of the team and should allow team members to use it effectively and successful. According to King [1], "If health information is to be effective, it must be communicated in such a way as to motivate each individual to understand it and then to use it". Effective collaborative communication is essential for the success of any endeavor. The Merriam-Webster dictionary defines effective as producing a desired effect and ineffective as not producing an intended effect. In the field of nursing, effective communication is considered communication that is precise and concise with a successful outcome. King's conceptual definition requires clear transactions between sources for effective communication to occur [2]. Research regarding communication between team members in hospice interdisciplinary team meetings, communication between physicians regarding hospice and palliative care, technology that can improve both communication and collaboration between team members has been reported. Hospice has been defined by the National Hospice and Palliative Care Organization (NHPCO) as "hospice care involves a teamoriented approach to expert medical care, pain management, and emotional and spiritual support expressly tailored to the patient's needs and wishes. Support is provided to the ?patient's loved ones as well. At the center of hospice and palliative care is the belief that each of us has the right to die pain-free and with dignity, and that our families will receive the necessary support to allow us to do so [3]. Additionally, measures for collaboration and communication have been identified. However, there is limited data regarding effective collaborative communication between clinical and non-clinical team members in (a) hospice admissions settings. The purpose of the study was to explore communication between clinical and non-clinical team members in a hospice (home) care setting and specifically, the admissions department. A component of this project involved efforts to develop a survey for data collection. The survey was a test survey instrument that was being developed for future research on the collaborative communication between the clinical and nonclinical team members in the hospice admissions setting. The initial efforts undertaken as a preliminary step of the broader objective to learn more about communication between clinical and non-clinical communication of hospice admission teams, focused on instrument development, pilot testing of the instrument tool and analysis of the reliability of the instrument.

Perceptions of communication between clinical and nonclinical hospice admission team members for the sample participants were analyzed to glean a better understanding of their perceptions of team communication and garner information that can foster instrument development of the survey tool.

\section{Theoretical foundation}

The theoretical basis for the research project was Imogene King's middle range Theory of Goal Attainment. The theory focuses primarily on the interpersonal system as well as the interactions that take place between individuals, specifically with the nurse patient relationship. The nurse's relationship to 
practice would be the nurse functioning through interactions with individuals and groups and within the environment [4].

King's Theory of goal attainment is considered a middle range theory [4]. King's theory focuses on improving personal interactions. King and Fawcett [5], stated that human beings are open systems who set goals and who select means to achieve them. King indicated that open systems exchange information and energy and are goal-directed. King's theory is based on an assumption that human beings are the focus of nursing. She states that the goal of nursing is health: 1) its promotion, maintenance and restoration; 2) the care of the injured or sick; and 3) care of the dying [6]. In the early 1960's, King discussed the need to focus on and organize all existing knowledge is nursing as well as expand the overall knowledge base for the nursing field.

King [1] published a conceptual framework for nursing focused on personal, interpersonal and social systems and now expanded to include information, communication, energy, social organizations, interpersonal relationships, status and role. King [2] also tested the Theory of Goal Attainment to describe the level of support that varied from her previous study and stated that her middle range theory described the nurse-patient interaction process that would lead to goal attainment (Sieloff \& Messmer, 2010). To determine if nurses made transactions, King's research described a process that led to goal attainment and studied the nurse-patient interaction process on a patient care unit in a hospital setting. King developed a goal-oriented nursing record that could assist nurses to determine if they were making adequate transactions for goal attainment. This process was called a model of transaction.

King viewed individuals as open systems with an exchange of internal energy that was external to human beings (Sieloff \& Messmer, 2010). Nurses communicate primarily with other individuals and both in and out of practice. This makes King's Theory of Goal Attainment a very practical nursing theory. Effective and collaborative communication had been suggested as a research topic by the author to improve processes and communication among all team members. Clinical and nonclinical team members are in constant interaction with individuals and groups in their environment including each other.

Additionally, there were two collaboration models that have been reviewed as research for this study. Kuebler \& Bruera [7] developed a standardized communication format called the Collaborative Consultative Model (CCM). The model provides practioners that collaborate with a standardized communication form. The form contains various psychometric assessment instruments that can be accessed via Internet. There are advantages and disadvantages of the CCM. The advantages include: the opportunity to provide expertise worldwide through the ease of the Internet, educational and clinical support any provider that is not familiar with palliative interventions, the model can provide a greater database for research, the number and type of assessment tools can be negotiated between collaborators, and the model can provide expertise in remote areas worldwide. The disadvantages of the CCM include: possibility of legal ramifications between countries and states, ethical considerations, time responsiveness, reimbursement issues, international differences, cultural differences, and censorship between individual practitioners and the consultant. The model has been developed between a nurse practitioner and a physician that collaborated on a few patients. Kuebler and Bruera [7] stated the CCM has potential to support evidencedbased palliative care for clinicians. The process would take place through a standardized plan to improve clinical outcomes as well as patient and family satisfaction.

After reviewing multidisciplinary theoretical literature, conceptual concepts and research concepts from social work literature, Bronstein [8] developed a two-part model for interdisciplinary collaboration. The first part of the model consisted of five components necessary for collaboration between social workers and other disciplines that ?included: flexibility, collective ownership of goals, interdependence, professional activities that may be newly created and the reflection on the process. The second part of the model consisted of four influences on collaboration that included: structural characteristics, professional role, personal characteristics and a history of collaboration. Bronstein [8] stated that interdisciplinary collaboration maximizes the expertise that each discipline can offer and is critical to colleagues working as a team. Interdisciplinary collaboration would minimize the need to continually redefine the process of collaboration.

Following a systemic review of the literature, it was determined that there is a lack of literature on collaborative communication between team members in a hospice admission team. King's middle range Theory of Goal Attainment provided the guiding framework in exploring the process of communication and interaction. The research regarding effective collaborative communication between clinical and non-clinical admissions team members in a hospice setting is limited. There is research evaluating the interdisciplinary team (IDT) as well as health care units but the evaluation of hospice teams and interactions outside of the IDT were very minimal. Demiris et al. [9] verified that teamwork and interdisciplinary collaboration are necessary for efficient health care services. Furthermore, Bushinski and Cummings [10] stated ineffective end-of-life communication with patients and families may lead to futile use of resources as well as futile medical care. Effective communication and collaboration between interdisciplinary team members is essential. Measurement of effective communication and collaboration presented a challenge in this instance. Established instruments were not available. To address this limitation, a survey tool was developed and piloted as part of this project. Refinements of this preliminary instrument will contribute to future research efforts to evaluate communication between interdisciplinary team members. Ultimately an investigator's ability to determine if collaborative communication was indeed occurring and verify the perception of communication and collaboration between team members, will positively contribute to patient care in a hospice setting. Furthermore, accurately validating if members of the team were comfortable in their role and felt comfortable 
asking for assistance within the team is expected to facilitate team performance.

\section{Literature Review}

A literature search undertaken to find data related to the topic of effective communication patterns for clinical and nonclinical team members in hospice admissions setting yielded scant results. Cinahl was the primary search engine used with the phrase 'effective communication between employees in hospice' searched. There were also several key words utilized in the search. They included: team, interdisciplinary, collaboration, communication, effective and ineffective communication, cooperation and hospice teams. Studies that have addressed communication in the hospice setting focused on communication in the interdisciplinary team meeting setting. There were 10 articles found with the words communication and teams however they focused on meetings. There were three articles that addressed teams and communication outside of the interdisciplinary team meeting. There were 20 articles that focused on palliative care and communication in various settings. There were also multiple international articles that 7 dealt with hospice and palliative care in the inpatient hospital setting. There were no specific articles regarding effective communication between clinical and non-clinical employees in a hospice admissions setting. Studies on communication and communication styles have been published however articles that focused on communication and collaboration between team members in hospice care outside of the IDT were not found in published referred journals.

Corcoran and Casarett [11] reported that end-of-life often means uncomfortable conversations. There is little research regarding when conversations should take place but the authors acknowledged that end of life conversations are crucial. The authors reported that even the best conversation or information may not ensure that hospice patients will enroll in hospice in a timely manner. Hospice and palliative patients have a right to know about their health and their prognosis. The authors suggested that patients at the end-of-life should have access to treatments supported by medical evidence and that could potentially improve their quality of life. Corcoran \& Casarett [10] stated that communication can improve end-of-life care but the care should also be aligned with the patient's and family's goals and need for care. Communication in hospice and palliative care is necessary and should always be a part of the plan of care.

Dunne [12] discussed communication in palliative care as a transactional process that can potentially affect the patient, family and nurse. The purpose of the author's qualitative study was to increase nurses' awareness of the communication process and to encourage them to reflect on their own practice to improve communication skills. The author identified the nurse-patient relationship as a central point in communication and the relationship. Patients have fear and anxiety regarding the dying process therefore communication becomes a critical and essential element. Nurses experience stress and difficulty in caring for palliative care patients. Dunne identified that nurses often block effective communication by blocking and distancing tactics such as avoiding emotional conversations. The nurses' behavior may be an effort to avoid uncomfortable conversations or protect emotions. Dunne [12] concluded that it is important for nurses to develop communication skills that can assist them in interpersonal contact with patients as well as others. Interpersonal contact eases the stress that is caused by miscommunication in health care between patients, families and providers. Effective communication can enhance the dying process.

Patient's perceptions of their end-of-life needs were recently addressed by Arnold [13]. His investigation used an exploratory mixed methods design, to obtain data on the patients self-report of their needs when coping with a terminal illness or disease process. Arnold found that the needs of critically ill individuals are multi-dimensional. The needs identified included: time, social, safety, physiological, death and dying, spirituality and change and adaptation. Arnold also indicated though that patient's needs are often ambiguous and uncertain due to the inability to specify which needs are not being met and he suggested that communication from health care providers is the key to optimal palliative care.

The study revealed several key points. There is an opportunity to develop sensitive clinical communication-assessment tools that use language to accommodate the patient.

Arnold [13] also stated that the findings suggesta starting point for development of a 9 palliative care-specific theoretical model. This model would create a deeper and holistic understanding for end-of-life needs. Collaboration and communication regarding end-of-life issues can assist palliative patients and families to redefine needs.

Dale [14] discussed the effectiveness of adequate communication in an editorial. As a third-year nursing student, the author discovered that it is easier to communicate with patients when a relationship is built that is not just a clinical relationship. The investigator identified the emotions that patients may be feeling which included anxiety, confusion and worry. Additionally, she advised that providing emotional support, information and reassurance facilitated effective communication. The author concluded that nurses must remain confident to communicate effectively with their patients. This will help ensure optimal clinical care by allowing patients to feel more comfortable and enable open communication. Furthermore, evaluating communication and patients' perceptions increased awareness for the student. Dale's opinion was noteworthy as it was a commentary from a student's perspective and it may also assist in understanding various perceptions.

In a similar theme, Bushinski and Cummings [10] stated that end-of-life conversations are essential to ensure that patients and families' goals, values and care plans are met. Bushinski and Cummings [10] stated that the manner in which the conversations took place as well as the timeliness of the conversations were both important factors. Additionally, ineffective end-of-life communication with patients and families may lead to futile use of resources as well as futile medical 
care. End-of -life conversations, though difficult, are essential to successful outcomes and adequate clinical 10 care. However, Blinderman and Prager [15] stated that health care professionals do not communicate well. Professionals do not adequately assist grieving families to cope with death and dying. Blinderman and Prager stated health care professionals received little training to cope with discussions about death and dying but palliative care specialists and medical educators were able to teach skills of active listening. The authors suggested that physicians and other medical personnel can be taught active listening to prevent futility in medicine by discussing death and dying. Blinderman \& Prager stated that clinicians can refine their communication skills and abilities and optimize patient preferences when they are properly mentored and trained. Additionally, Blinderman and Prager [15] demonstrated that active listening skills can decrease the medical liability for all health professionals if active listening skills were developed.

Betcher [16] focused on developing a nurse's confidence and communication ability when engaging in emotional discussions with patients and families. An eight hour education program on palliative care involving role-playing and simulation was created to assist nurses to communicate effectively with endof-life issues. The author developed the "Elephant in the Room" project to improve communication skills of nurses to help them communicate effectively and compassionately with palliative patients and their families. Nurses in this study were able to apply new knowledge to their already existing knowledge of palliative care. The project allowed nurses to practice communication scenarios through role-playing and simulations. Nurse's communication should be a holistic perspective and would be a greater benefit to patients and families.

Clarke and Ross [17] surveyed the perceptions and communications of nurses working with the elderly and with patients receiving palliative care. The study was pivotal in assessing factors influencing nurse's communication with the elderly at the end-of-life. Many factors effect communication. Included among those that are consequential with respect to end of life care are: nurse's perceptions; experiences of listening and talking to older adults; and learning from various members of the hospice team. Organizational and environmental constraints such as privacy, time and culture of care; perceived differences between values of nurses, physicians, patients and families were also found to influence communication. All participants of the study recognized the importance of communication with older adults at the end-of-life. Nurse recognized that discussions regarding prognosis as well as involving older adults in decisionmaking were essential. Additionally, Clarke and Ross [17] discovered that end-of-life communication required support from the organization or institution by using experienced nurses, engaging in multi-professional teamwork as well as doctors acting as role models. The authors claim all these factors must be present for nurses' to be fully effective in improving end-of-life outcomes.

Ashurst [18] discussed effective communication in palliative care. The author indicated that staff in residential centers needed to listen to residents openly and without judgment to assess their pain and their dying concerns. Residents should also be given the opportunity to speak with their relatives in a quiet and private area that is away from other residents to promote open communication. Ashurst stressed the importance of informed choice for residents and indicates that residents should be able to choose how to 12 spend their last weeks. The main suggestions offered to maintain effective communication were to listen openly and attentively and listen without offering advice. Additionally, the author stated that residents in residential centers needed to be supported and encouraged so they felt comfortable expressing their feelings. The author emphasized that communication should be transparent and honest to develop a trusting relationship with the patient and family. Empathetic listening, honesty, and support were all important components for effective communication.

Malloy et al. [19] used a qualitative study to investigate communication skills of nurses in palliative care. Effective communication is a key component in palliative care and is often considered the foundation to build a relationship with the patient and family. The investigators proposed that nurses providing palliative care must be trained in skillful communication. To further explicate what aspects of communication were of concern to nurses providing end of life care, the authors distributed a survey to nurses attending one of five End-of-Life Nursing Education Consortium (ELNEC) programs. The ELNEC programs were intended to prepare nurses on pain management, end-oflife issues, ethics, grief, and communication. The survey results indicated that nurses found discussing palliative care issues on religion, spirituality and talking to patients after they received bad news were reported as very difficult aspects of communication. Furthermore, nurses identified that communication with patients and families from different cultures were the most difficult aspects of communicating in hospice care with patients and families Malloy et al. [19].

The authors concluded that nurses will continue to play a critical and pivotal role with 13 communications in palliative care. Training and education may be required for nurses to communicate with their patients and their families effectively. Understanding and expressing therapeutic communication in hospice and palliative care is necessary and should be emphasized in nursing programs at the graduate and undergraduate level as well as a part of professional develop.

\section{Collaboration in a Team Setting}

Lyles and Oliver [20] studied interdisciplinary team (IDT) collaboration and communication in a hospice setting. The investigators used a mixed design incorporating qualitative and quantitative methods. IDT meetings are a forum for interdisciplinary collaboration between team members that allow open communication about patients and families in a professional setting. The purpose of the study was to identify collaborative communication as well as extend the theoretical framework of interdisciplinary collaboration in a hospice setting. Lyles and Oliver [20] defined effective IDT's as a positive climate, a shared language among staff from various disciplines, and team 
leadership provided by various disciplines to attain the ultimate goal of interdisciplinary collaboration. The team consisted of 8 team members in attendance with the meeting approximately 1.5 hours in duration. Ethnographic fieldworks of five (IDT) meetings in a hospice setting in the western part of the United States were studied. The teams were divided by geographic location into east and west teams as well as location such as home or nursing home with each IDT meeting consisting of a team leader (nurse) and a medical director. A triangulated approach was utilized to reveal evidence-based examples of collaborative communication to make the study more realistic.

The independent variable in this study was communication; the dependent variable was the interdisciplinary team collaboration. Discussion of the patient's case between team members was the unit of analysis. Each staff member also completed the Modified Index of Interdisciplinary Collaboration (MIIC). This tool was based on the same conceptual framework as the Index for Interdisciplinary Collaboration and thus was assumed to have the same validity. Observation of the IDT meetings suggested that collaborative communication was sustained through four different types of communication: 1) interdependence and flexibility of the job; 2) newly created professional activities; 3) collective ownership of goals; and 4) a reflective process. Case managers were the team members most actively involved with collaborative communication. The overall mean for the instrument was 1.91. With the MIIC, 1.0 was the highest perception of collaboration and 5.0 was the lowest perception of collaboration. The team ranked high on perceived interdisciplinary collaboration. The qualitative and quantitative findings suggested that collaboration occurs inside hospice as well as outside hospice. The qualitative analysis indicated that hospice interdisciplinary collaboration included flexible work roles that allowed IDT members to collaborate with non-hospice staff outside of IDT. The study conducted by Lyles and Oliver [20] revealed that both qualitative and quantitative findings suggested that interdisciplinary collaboration also occurs outside of hospice with professionals such as physicians and nursing home staff. The study will assist the authors study to evaluate effective collaborative communication between clinical and non-clinical employees in a hospice setting. Although the study by Lyles and Oliver [20] specifically discussed IDT teams, collaborative communication is imperative in all settings in hospice between team members. The authors discussed the power of interdisciplinary collaboration in hospice between team members in a hospice IDT meeting.

Demiris et al. [21] studied the information flow of hospice interdisciplinary meetings and focused on information access, documentation and exchange of information between team members in a qualitative and quantitative study. They verified that teamwork and interdisciplinary collaboration are necessary for efficient health care services. The authors studied actual meetings to gain understanding of information flow during the hospice IDT meetings. The authors investigated various members of four interdisciplinary hospice team meetings in the Midwestern United States. The interdisciplinary team included nurses, physicians, social workers, chaplains, volunteer coordinators, home health aides and bereavement counselors. Important findings from their research included the following: defining a leader or facilitator to increase efficiency, ensure access of patient charts for all team members during IDT, structured documentation of patient care discussions, information technology (IT) utilization for team members unable to attend the meeting, dynamic design and structure of teams for ongoing evaluation and revision, and the design of the meeting space as it can impact the effectiveness of the team. The authors reported that teamwork requires a detailed communication strategy that establishes effective sharing and flow of information. The authors concluded that the improvement of interpersonal communication requires an analysis of interactions among hospice team members and the ability to identify possible barriers to information flow, including the need to study interactions among team members.

Wittenberg-Lyles et al. [22] studied backstage communication in IDT meetings. Backstage communication was defined as the communication between team members that takes place away from patients and families. Each team member individually spent their work day visiting patients and families to provide needed services. The IDT provides an opportunity for the team members to collaborate on patient care goals and interventions. They met backstage apart from the other services that were provided. Team members also used the meetings as a place to share the emotional dimension of their work. The study confirmed that nurses dominated the meeting communication time delivering medical information as well as offering clinical impressions concerning the patients well being. This finding may be attributed to the schedule and nursing is the discipline that sees the patient most frequently. The authors concluded that backstage communications in hospice IDT meetings enable hospice staff to manage emotions safely backstage and prepare for frontstage professionalism. The IDT meeting and backstage communication allowed an opportunity for team members to bond and discuss their own emotions with their team.

Wittenberg-Lyles et al. [22] conducted an exploratory study of interpersonal communication in hospice IDT meetings. The purpose of the study was to explore relational communication control used by IDT members to share information and contribute to decision making. There were several conclusions gleaned from the study. First, providers should reflect on their own practices and behaviors in IDT meetings. There should also be discussion to the type of information that is considered the standard discussion in the meeting. Next, providers should encourage other team members to share information during IDT meetings. It is imperative to include all aspects of the patient's health to include the physical, psychological, spiritual and emotional issues with the family and patient. The authors indicated that best practices should be explored to enhance communication. Finally, providers should compare how interpersonal communication in team meetings would assist in development of care plans. The authors concluded that interdisciplinary communication was necessary to develop appropriate care plans and to include all members of the team. 
Demiris et al. [9] studied technologies to support end-of-life care. The purpose of the study was to evaluate the current level of information system utilization in hospice and palliative care. It was discovered in the study that caregivers rate communication with caregivers as essential to the support they receive during contact with various providers in hospice care. The authors revealed that there are few informatics interventions designed for hospice and palliative care.

Demiris et al. [9] conducted a study to assess hospice IDT meeting team member preparation for collaboration in meetings. The purpose of the study was to assess how team members are trained for IDT and to assess the IDT meetings. There were 145 hospices that participated in a telephone survey.

The survey found that the majority of hospice agencies provided the necessary training to 18 individuals but did not provide team training. Training also took place only in orientation. The authors suggested that hospice agencies needed to allow time for teams to engage in team-building activities. IDT meeting training should include preparation for the structured format of IDT meetings as well as any informal communication that may occur. The authors also suggested that nurses who lead meetings should be trained on how to involve all team members rather than expecting individual team members to share information when it is appropriate to do so. The authors concluded that training should be more consistent and focus on creating positive communication strategies to maximize the benefits of IDT meetings.

Ponte et al. [23] suggested that interdisciplinary teamwork and collaboration is an essential element of a positive practice environment. The authors further suggested that more research should be done to assess the impact of various leadership and organizational structures and its influence on advancing both teamwork and collaboration. The authors concluded that the team leader or facilitator is just as a large part of collaboration as any other team member.

Interdisciplinary collaboration and communication is also essential to successful hospice and palliative care. Austin et al. [24] determined that collaborative research requires diverse teams, shared space to meet, shared objectives and a strategic plan. Collaborative teams require a sense of safety, respect and trust. These qualities were described as essential to effective interdisciplinary communication. The authors cautioned that various disciplines may interpret different meanings for the same word. They suggested that accessing a broad base of expertise in interdisciplinary teams 19 can be beneficial. Various viewpoints are necessary to acknowledge all aspects of a situation.

Interdisciplinary collaboration in hospice involves the patient and family in theory. McDonald and McCallin [25] suggested that interprofessional collaboration supports patient-centered care and is parallel with teamwork. The authors questioned how families could be the center of care if they are not included in the collaborative process. Patients and families may not have the time required to participate in the interdisciplinary collaboration because of the demands on their time that occur when an individual is in hospice care however they should have the opportunity to participate if able. The authors also stated that interprofessional collaboration encourages communication through interaction with team members and sharing of knowledge. They emphasized that understanding the different roles was essential to success. Education for health care workers was also imperative for the process to be successful. There is agreement that understanding the interpersonal skills of other team members is necessary for collaboration in clinical practice. Kay et al. [26] not only acknowledge the value of education in team members to ensure collaboration but also indicated that lack of multidisciplinary relationships is a factor in the failure of integration of palliative care. Education and building effective multidisciplinary collaborative relationships with peers is essential for collaborative communication.

There must also be an understanding of the actual definition of interdisciplinary collaboration for the concept to be truly integrated into practice. Petri (2010) explored the meaning of interdisciplinary collaboration within the context of health care. The author stated that shared decision-making was necessary for interdisciplinary collaboration to take place. Interactional skills are also required and include mutual respect, effective communication and trust. To promote successful interdisciplinary collaboration into healthcare delivery, the author suggested the following as necessary factors: trust; mutual respect; open and effective communication; awareness and acceptance of roles and skills; understanding responsibilities of the participating disciplines. Interdisciplinary collaboration will continue to be defined for use in future practice.

There has been research regarding collaboration in health care units that affirmed previous findings. Weinberg et al. [27] examined collaboration among interdisciplinary providers on health care units. The authors suggested that leadership is important for collaboration and teamwork to occur and that in the absence of formal teams, leadership is essential to provide feedback and respond to employee needs. Collaboration and teamwork follows a hierarchical pattern based on occupation, education, salary and ability to make decisions. Providers that were at the top or towards the top of the hierarchy perceive their environment more positively than those in a lower hierarchy. Success in attaining collaborative interdisciplinary teamwork may not be possible for all hospitals. The authors stated that though tasks in hospitals are interdependent, providers may have little contact with each other and would not consider themselves as members of a team. Collaboration and teamwork must take place simultaneously to be effective in the hospital setting. To ensure better patient outcomes, team members must communicate collaboratively and effect.

\section{Technology and Collaboration}

Green and Thomas [28] studied the importance of interdisciplinary collaboration and the electronic medical record (EMR) and physician's perceptions of nursing documentation after EMR implementation. To effectively practice medicine, physicians request detailed assessments and interventions in 
nursing documentation. The detailed assessments assisted their own practice as it was more informative and the information was clearer. The authors concluded that physicians desire nursing documentation with greater clarity and narrative summaries. Joint collaboration is necessary to develop effective EMR systems.

Another study revealed that technology can improve communication. Dy et al. [29] identified that routine electronic patient-reported outcome collection in patients with advanced disease could improve communication among caregivers, patients, and providers as well as the timeliness of identifying problems and the effectiveness of follow-up. The authors developed a web-based tool to collect symptoms and data needed for hospice patients and families. Information technology (IT) would focus on development to facilitate hospice processes. The creators believed hospice providers would only use the system if they felt it would be useful in improving clinical care. The authors concluded that providers, organizations, patients and caregivers will not use a system unless it improves their clinical practice or quality of care. It is imperative that a clinical system is identified as a user-friendly system to ensure that the product is utilized effectively.

Shirren and Phillips [30] conducted a study to understand the use of technology to support interpersonal interaction. Thirtynine employees completed a five-day communication diary recording their actual behavior when receiving personal and work-related e mails. The authors found that higher numbers of work-related e mails were related to higher levels of stress. Higher levels of negative affect correlated to a delay in dealing with personal and work e mails. Suggestions were made by the authors to provide systems that sort and prioritize e mails to use e mail more efficiently and reduce the number of low-quality e mails. Educating employees on responding to e mails in an adaptive manner was also suggested. The authors concluded that while technology and information is essential, too much may be detrimental to productivity.

Many hospices now use electronic medical records (EMR's) as a part of their daily routines. Since the majority of the staff makes visits to patient's homes, it is essential that the technology is simple to use and consolidated. Hospice team members often receive more e mails and messages that a traditional hospital setting. The optimal utilization of technology would be for the coordinator to only send messages if they are vital or specify certain times of the day when messages that are not significant may be sent.

\section{Measures of Collaboration and Communication}

Dawson [31] conducted a study to analyze lack of collaboration between teams. The goal was to improve collaboration and communication in order to promote an effective working environment and to create effective interprofessional relationships. As a community Clinical Nurse Specialist (CNS) at a hospital in Manchester, United Kingdom, the author served as a liaison between palliative care teams that worked independently of each other. The role of the community CNS would be to attend the multidisciplinary team meeting at the hospital and then report back to the community palliative care team. Some barriers to collaboration between the hospital and the community that were identified early included: staff constraints, lack of time, heavy workloads or complex patients. While the goals and commitment that the palliative care teams showed towards their patients were similar, they did not create the time for proper team building. Team-building sessions were thought to be beneficial but would require time and a facilitator. Furthermore, establishing team building sessions would also require team members to concede territory, cross boundaries and accept diversity. Teaching opportunities were presented to all palliative care teams to bridge the divide between teams and to gain various perspectives and expertise on subject matters. After a period of 18 months, there were improvements in collaboration and communication between the teams. The introduction of the CNS to facilitate communication and collaboration was beneficial. Dawson [31] identified several factors that contributed to effective collaboration between teams including: self-analysis, flexibility and empathy that the author achieved through listening, inquiring and shadowing. Recommendations for future collaboration included: the development of monthly or bi-monthly interprofessional meetings involving both palliative care teams to sustain collaboration; individual members of the team to shadow their colleagues in practice; and finally to continue joint educational sessions to promote sharing of perspectives as well as providing insight into practices which may be invisible to outsiders. Dawson [31] also suggested that to sustain collaborative teams, more work is required and work should focus on team-building activities with individual members with a purpose to reduce barriers and embrace diversity.

Thannhauser et al. [32] reviewed quantitative measures described in the intraprofessional literature to evaluate collaborative practice. Twenty-three instruments were identified and analyzed for reliability and validity of the instrument, ease of access to items on measure, sample size and applicability to diverse professional populations. Health care professionals were expected to communicate collaboratively in order to provide effective care to patients and families. The authors evaluated interprofessional collaboration (IPC) among individuals rather than teams. The authors specifically hoped to identify attitudes that individuals had towards IPC as well as their ability to collaborate in the field of obesity prevention, research and treatment. There were not specific measures that could be used for the purposes of this investigation; however the information was valuable in the initial effort undertaken to develop an instrument for use in measuring interdisciplinary collaboration in the hospice setting. A key point noted by the investigators was the lack of adequate tools for assessing IPC. The authors also suggested that IPC is a necessary component to advance health care and improve the quality of health care and social services. It is also important that health care professionals agree on the definition of IPC and the elements necessary for collaborative learning and practice. Further research is needed to develop quantitative measures for collaboration.

Junger et al. [33] focused on factors for successful multiprofessional cooperation from the perception of team 
members in a palliative care unit. The study had two primary purposes: to provide a systematic inventory of factors necessary for team work in palliative care and to identify criteria that enhances the success of multiprofessional cooperation within a palliative care team. The qualitative study emphasized team member satisfaction, team work and organizational commitment. Team member's defined several factors necessary for cooperation in a palliative care team. They included: communication, work climate, team philosophy and team commitment, flexibility and openness. Ineffective cooperation was also defined by the team and included ambiguity of goals and procedures, lack of communication as well as task conflict. The authors also suggested two key factors for the development of new palliative care teams. First, it is important to maintain structure, clarity and establish realistic expectations. Secondly, goals and expectations should also be clear and adequate time for exchange of information should be allotted.

Campion et al. [34] explored how to design successful work groups. The authors found that job design characteristics may be useful in predicting effectiveness and interdependence characteristics may also have some value. Most importantly, the authors suggested that communication between work groups might relate to productivity in groups with highly interdependent tasks. Communication plays a key role in team dynamics and may improve productivity.

Curry et al. [35] identified an increase in productivity when team members worked together collaboratively. The purpose of the study was to analyze interpersonal interactions of an interdisciplinary research team as well as actions taken when conflict arose within the team. When conflict interrupted the teams work and collaboration, the principal investigator would call a meeting and a discussion ensued regarding reasons for the turmoil. Research teams are often in conflict. There are many educated professionals with strong views on several topics that they are passionate about. Collaboration among team members was achieved when members thought aloud, when they offered solutions for each other, when they expressed gratitude and when they were respectful to other team members. Trust became not only a prerequisite but also an outcome of the process. The authors stated that collaboration was enhanced by negotiating politics of identity, improving communication among team members, building trust within the team, and valuing the diversity of all members. Collaboration between team members can be achieved when trust is a prerequisite and can also increase team productivit.

\section{Instruments}

McCaffrey et al. [36] conducted a quasi-experimental study to determine the effect of an educational program on nurses and medical resident's attitudes toward positive collaboration and communication. A weekly follow up meeting was a component of the project. The study was conducted at a South Florida hospital in 2008-2009 when a new residency program was set to begin. The nurses had no prior experience in working with medical residents. The study used the Jefferson Scale of Attitudes towards Physician-Nurse Collaboration which is a 15 question
Likert scale and the Communication, Collaboration and Critical Thinking for Quality Patient Outcomes Survey tool which is a 13 item questionnaire also using a Likert scale. The purpose was to measure the attitudes of 47 residents and 68 nurses with positive communication and collaboration. There was a significant difference in the pre and post test scores for the nurses and the residents. The findings indicated that there was improvement in the use and understanding of effective communication skills. Both scales had been previously tested for reliability and validity; however, the scales were found to be complex, time consuming and difficult to understand.

Communication in palliative care and hospice and interdisciplinary teams has been the focus of numerous investigators. There is literature to support technology and the impact on collaboration as well as measures of collaboration and communication. The intent of this project is to gather information on communication between clinical and non-clinical team members in a hospice setting.

\section{Methodology}

The research question posed toaddress the focus of this project was: What are the perceptions of the clinical and nonclinical admission staff members concerning their communication with each other relative to patients who are being admitted to a hospice care environment?

\section{Study Design \& Sample}

A descriptive design was used to gather data from hospice employees on the perceptions of effective and ineffective communication (E/IC) between clinical and non-clinical team members in a hospice admissions program. The project also incorporated efforts to develop a survey instrument that was used to gather information from the participants. Approval from the University Internal Review Board (IRB) was obtained and from the cooperating hospice agency director.

The target population was all clinical and non-clinical team members who provide hospice care. The design incorporated a convenience sample which included clinical and non-clinical team members in a hospice admissions setting. The sample selection was obtained by inviting all of the team members in the hospice admission department to an explanation of the project goals and a review of the risks and benefits of the study. Potential participants were given an opportunity to ask questions. Inclusion criteria for the study were (a) hospice team member of the admission team, (b) team members must have history of communicating with each other, (c) team members were English speaking. The exclusion criteria for the study were (a) nonEnglish speaking employees, (b) team members that are not on the admissions team (c) volunteers of hospice.

There were 19 potential eligible participants. All eligible staff members voluntarily choose to participate in the survey. Each participant was given a consent form. Following completion of the written consent form, a copy was provided to the study participant and contact information to reach the investigator was made available. 
Table 1: Demographic Table.

\begin{tabular}{|l|c|c|c|c|}
\hline $\begin{array}{l}\text { Demo } \\
\text { Table }\end{array}$ & Frequency & Percent & Valid Percent & $\begin{array}{c}\text { Cumulative } \\
\text { Percent }\end{array}$ \\
\hline Female & 14 & 73.7 & 73.7 & 73.7 \\
\hline Male & 5 & 26.3 & 26.3 & 100.0 \\
\hline Total & 19 & 100.0 & 100.0 & \\
\hline
\end{tabular}

Table 2: Team Table.

\begin{tabular}{|l|c|c|c|c|}
\hline Team Table & Frequency & Percent & $\begin{array}{c}\text { Valid } \\
\text { Percent }\end{array}$ & $\begin{array}{c}\text { Cumulative } \\
\text { Percent }\end{array}$ \\
\hline Clinical & 8 & 42.1 & 42.1 & 42.1 \\
\hline Non-Clinical & 11 & 57.9 & 57.9 & 100.0 \\
\hline Total & 19 & 100.0 & 100.0 & \\
\hline
\end{tabular}

Table 3: Race Table.

\begin{tabular}{|l|c|c|c|c|}
\hline Race Table & $\begin{array}{c}\text { Frequen- } \\
\text { cy }\end{array}$ & $\begin{array}{c}\text { Per- } \\
\text { cent }\end{array}$ & $\begin{array}{c}\text { Valid Per- } \\
\text { cent }\end{array}$ & $\begin{array}{c}\text { Cumulative } \\
\text { Percent }\end{array}$ \\
\hline White & 18 & 94.7 & 94.7 & 94.7 \\
\hline $\begin{array}{l}\text { African } \\
\text { American }\end{array}$ & 1 & 5.3 & 5.3 & 100.0 \\
\hline Total & 19 & 100.0 & 100.0 & \\
\hline
\end{tabular}

Table 4: Sample Characteristics of participants $(n=19)$.

\begin{tabular}{|l|l|l|}
\hline Variables & Frequency & Percen \\
\hline $\begin{array}{l}\text { Gender } \\
\text { Male }\end{array}$ & 5 & \\
Female & 14 & 26.3 \\
\hline Race & & 73.7 \\
Caucasian & 18 & \\
African American & 1 & 94.7 \\
\hline Team & & 5.3 \\
Clinical & 8 & \\
Non-Clinical & 11 & 41.1 \\
\hline
\end{tabular}

The primary variable of interest for this study was collaboration between clinical and non-clinical team members. Information on collaboration was gathered through the use of a survey instrument. Demographic information was obtained for the purpose of describing the study sample and obtained through the use of dichotomous questions.

\section{Instrument}

The scale for data collection in this study was developed by the investigator. An extensive search was conducted to find an appropriate tool. An instrument was not identified that was specifically developed to describe elements of collaborative communication between hospice team members. The areas of focus for this project were the perception of communication and collaboration between team members, if members of the team were comfortable in their role and also felt comfortable asking for assistance within the team.

A Likert type scale was constructed using eight declarative statements. Respondents had a range of bipolar ratings to indicate their degree of agreement or disagreement with the statement. The end points of the scale provided the extreme opposites on the continuum of the scale. The Likert scale format was chosen because it is a traditional scale based on classical measurement theory.

Positively worded statements were constructed at a $7^{\text {th }}$ grade reading level. Short sentences were used to promote clarity. The items selected for the declarative sentences were based on the literature review articles that specifically addressed communication and collaboration and directed toward three components of hospice team interaction. They are communication, resources and roles and thirdly collaboration. The first four items of the scale formed a sub-scale on communication. Two items addresses the adequacy of resources available to the clinical and non-clinical staff and the perception of the participants regarding the rolls of the various team members. Two items were constructed for collaborative interaction, forming a third sub-scale. The full eight item scale was estimated to take approximately 10-15 minutes to complete. A section of the 30 survey was created for the participants to add their own comments if they choose to do so. The scale is identified as the Communication and Collaboration Rating Instrument (CCRI). A rotated factor matrix was completed for validity.

\section{Data Collection}

The survey was conducted prior to a team meeting of the hospice admissions staff. Clinical and non-clinical staff members were participants. All participants completed the survey at the initial data collection session. The surveys were then placed in a blank, sealed envelope for confidentiality. The participants returned the blank envelopes to a sealed box that had a slot to insert the surveys. The setting for data the collection was a conference room at a hospice inpatient site in the Midwest. The hospice facility cares for patients and families in their homes (coordinated by team members in the office setting) and has an inpatient unit inside a nearby hospital. The data collection took place in the inpatient location of hospice unit.

\section{Demographics}

A total of 19 individuals completed the survey. Demographic

Table 5: Rotated Component Matrix.

\begin{tabular}{|l|c|c|}
\hline Component & $\begin{array}{c}\text { Factor I- } \\
\text { Plans and Progress }\end{array}$ & $\begin{array}{c}\text { Factor II- } \\
\text { Team } \\
\text { Commtyunication }\end{array}$ \\
\hline Daily Collaboration & 0.894 & 0.26 \\
\hline Sufficient Resources & 0.86 & 0.254 \\
\hline Team Support & 0.789 & 0.441 \\
\hline Team Communication & 0.587 & 0.369 \\
\hline $\begin{array}{l}\text { Strength/Skill of Team } \\
\text { Members }\end{array}$ & 0.525 & 0.903 \\
\hline $\begin{array}{l}\text { All Team } \\
\text { Communication }\end{array}$ & 0.78 & 0.878 \\
\hline $\begin{array}{l}\text { Communication } \\
\text { Quality }\end{array}$ & 0.121 & 0.837 \\
\hline Plans and Progress & 0.302 & 34.694 \\
\hline $\begin{array}{l}\text { \% of variance } \\
\text { explained }\end{array}$ & 37.067 & \\
\hline
\end{tabular}


data was limited to information on three variables, gender, race and the clinical or non-clinical employment status of the employee. The majority were White, $94.7 \%(\mathrm{~N}=18)$ and $73.7 \%$ $(\mathrm{N}=14)$ were female. The clinical staff participants comprised $42.1 \%$ ( $N=8$ ) of the participants while the non-clinical staff comprised $57.8 \%(\mathrm{~N}=11)$ of the sample (Appendix D, Tables 1-3).

\section{CCRI Scores}

The CCRI survey instrument contained eight items derived from the literature review. They were presented on a Likert scale with a possibility of five optional rankings. Scores provided data for analysis from 19 participants. Reliability coefficients were calculated on the total scale items as well as the subscales. The Cronbach's Alpha coefficient for the full eight items was .894. The sub-scale including four items on communication had a reliability coefficient of .893 . The reliability coefficient for second the subscale examining resources and roles was, .577 and the third subscale on collaboration was .789 respectively.

Further analysis was done to examine the relationship between the items of the scale. The item All Team Communication was strongly correlated with Plans and Progress, ( $r=.708$, p $<$ ).01); with Quality Communication, ( $\mathrm{r}=.783$, p, .01). Team Communication was also moderately correlated with Daily Collaboration, ( $\mathrm{r}=.490 \mathrm{p}<.05)$, Strengths and Skills, $(\mathrm{r}=.434$, $\mathrm{p}, .05)$, the ability to comfortably Ask Another Member for help, $(\mathrm{r}=4.35, \mathrm{p}, .05)$ and Team Communication, $(\mathrm{r}=.481 \mathrm{p}<.005)$.
The strengths of the relationships among the items prompted further analysis. Although the sample size was small, more than ten cases were generated for each of the items on the scale. Factor analysis was used to address the interaction of the items developed. Two statistical measures generated by SPSS provided assistance in evaluating the factorability of the data. Bartlett's test of sphericity indicated the significance was $<.05$, or $(.000)$ for the data. Secondly the Kaiser-Myer-Olkin Measure on sampling adequacy (KMO) was used to assess the adequacy of the sample for the appropriateness of factor analysis. The KMO value was .753 well above the minimum value of .6. The approach used was principle component analysis. The results yielded on the correlation matrix indicated all items were included. To further refine the development of the CCRI scale for the future use, factor rotation was used. Two benchmarks were used to determine the number of factors to include and which of the eight factors were appropriate to include. One criterion was that the percentage of shared variance by the factors included was equal to or above $60 \%$. The data can be reviewed in Appendix G, Table 5. Secondly the scree plot was examined. The scree plot demonstrated a sharp decline in the after the second component. Two items Plans and Progress, and Team Communication explained 71.76\% of the variance with respect to communication and collaboration. The scree plot (Appendix I, Figure 1) illustrated considerable discontinuity between the second and third factor, offering support for extracting the first two items of the instrument. Factor rotation, the second phase of factor analysis, was then

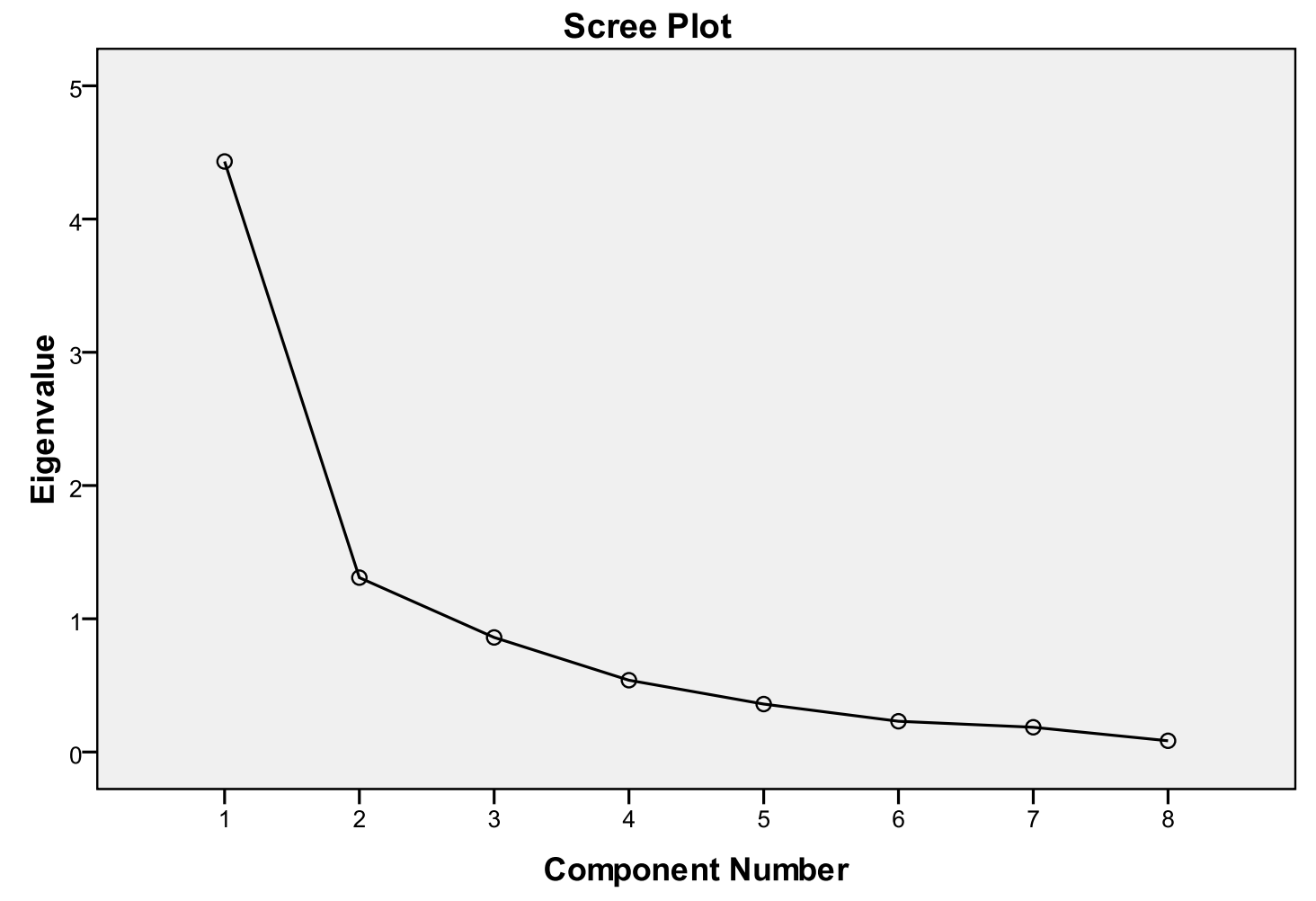

Figure 1: Screen Plot. 
used on the factors that met the extraction criteria. This was done to enhance the interpretability of the factors by determining what items clustered or was associated with a factor.

A rotated factor matrix, varimax rotation or the orthgoninal rotation (which assumes that the factors are not related) was used to aid in the interpretation of the findings (Appendix G). The loadings for Plans and Progress, or Factor I were substantial for five of the scale items, Daily Communication (.894), Sufficient Resources (.860), Team Support, (.789), Team Communication ( .587) and Strength /Skills of the Team Members (.525). The loadings for Team Communications, or Factor II were substantial of three of the scale items, All Team Communication (.903), Quality of Communication (.878) and Plans and Progress (.837). All eight items loaded substantially on one of the components. The two factor solution explains $37.067 \%$ of the variance for Factor I and $34.964 \%$ of the variance for Factor II, or $71 \%$ of the cumulative variance with respect to communication between clinical and non-clinical team-members. Results from this analysis support the future use of all eight items of the scale and suggests the reconsideration and revision of the sub-scales (Appendix G, Table 5).

Collaboration between clinical and non-clinical team members of the hospice admission team was of key interest at the initiation of this project. The sample was comprised of eight clinical members and eleven non-clinical members. The median scores for each of the eight scale items were examined for difference in the scores between the clinical and non-clinical participants. The Mann-Whitney U Test was used to analyze data. No significant differences were found between the two groups with respect to the perception of collaboration among the members of the admission staff.

However, a few trends are worth noting. The two items of the CCRI scale which reflected the highest consensus between the two groups were Strengths and Skills, and Plans and Progress. In both instances the respondents from both groups converged on the "strongly agree" response to the scale item statement: "I am well informed on the agencies plans and progress" and "The role of the admission team members are distributed according to the individual's strength and skills". The item "Team Support" which demonstrated a trend toward different perceptions stated "When I need assistance in helping a hospice patient or family member I feel comfortable asking for assistance."

The respondents from the clinical group ranked this item with a lower value than the non-clinical members whose responses indicated a higher level of agreement with the statement. Secondly, the statement "Communication among all members of the admission team is adequate", or "Team Communication" was ranked lower among member of the clinical group yet member of the non-clinical group trended toward agreement with this scale ite.

\section{Participant Comments}

A section of the survey was created for the participants to add their own comments if they choose to do so. The sample size limits a genuine qualitative analysis of the data; however it does provide valuable insight for future instrument development. Comments added by participants that were included in the item Plans and Progress, or Factor I including the following responses:

"Many things get missed because of so many e mails"

E-Mails get sent out the day before something is implemented".

The data while limited suggested that additional scale items that address the perceptions of technological communication are appropriate to consider in further development of the instrument.

\section{Results}

The participants who comprised the sample for this project are representative of the clinical and non-clinical admission staff at a Midwest hospice center as all members of the admission staff contributed data for analysis. The homogeneity of the sample provides some assurance that the data from this survey offers relevant information for participating agency to consider. For example, it is of value to note that all members of the admission team indicated that their perception was they were well informed about the plans and progress of the agency. This is positive and affirming feedback from both groups regarding the aspect of informational communication. Additionally, members from both groups, clinical and non-clinical, also reported that they perceived that the roles of the team members were distributed according to the individual's strengths and skills. This also provides constructive feedback for the agency that has the potential to indirectly influence patient care.

One item that demonstrated a trend toward different perceptions was "When I need assistance in helping a hospice patient or family member I feel comfortable asking for assistance." The respondents from the clinical group ranked this item with a lower value than the non-clinical members whose responses indicated a higher level of agreement with the statement. While the probability (p) is not significant at the .05 level, $p=.09$ for this item, the sample size may have masked the detection of a substantial finding and there is value in pondering the possible reasons for the trend. Alternative explanations might be considered for the divergence. The nature and function of the clinical team members may differ in many respects from the non-clinical team member. It is possible that the clinical staff function more independently with less need of assistance from others. If this were the case, one would expect to see the raw data reflecting a neutral response, or a direct disagreement. However, the respondent's answers clustered in the around the rank "agree" with two members (8\%) of the clinical participants indicating they "strongly agree". Among the non-clinical team members, eight $(72 \%)$ responded that they "strongly agree" with the statement that they felt comfortable asking others for assistance. The results suggest that non-clinical admission team members perceive teamwork is evident within this sub-group of the hospice admission team. It may be beneficial to further explore the comfort level in asking for help with the clinical members of the admission team to obtain a better understanding of the meaning of their responses. 
The statement "Communication among all members of the admission team is adequate" showed the greatest divergence in the responses when examined by groups.

The item was ranked lower among member of the clinical group yet members of the non-clinical group trended toward agreement with this scale item. The difference was not statistically significant, ( $\mathrm{p}=.08$ ) however a comparison of the percentage scores provide some information that may be worth further exploring. For example, $37 \%$ of the clinical team members perceived the communication among the team members to be adequate, while $72 \%$ of the non-clinical team members perceived that communication was adequate or a $35 \%$ difference in the responses on this item. Twenty-five percent of the clinical group disagreed that the communication among the admission team members was adequate; however no one from the non-clinical reported that their perception of communication among the admission team members was in disagreement with the statement.

Follow-up meetings of the hospice admissions team could provide an opportunity to discuss the finding from this initial project and explore what underlying factors contribute to the low percentage rate of clinical staff admission team members who agree that communication is adequate. Additional strategies to address the difference in perceptions might include a more open ended survey or providing a professional development education session for the entire team on communication.

15. Information gleaned from this study has limited generalizability with respect to the perceptions of clinical and non-clinical staff members in the sample to other agencies. Further studies are needed that included additional demographic information regarding the care providers by expanding the sample population to a more representative sample of the care providers in hospice settings.

\section{Reliability and Validity of the CCRI}

A Likert type scale was constructed using eight declarative statements. A range of bipolar ratings were used to indicate the participant's degree of agreement or disagreement with the statement. The end points of the scale provided the extreme opposites on the continuum with a range from 1-7. Items were developed bases on the literature and grouped together forming three plausible sub-scales. The high reliability coefficient calculated for these data reflects minimal error variance. The basic item analysis was accomplished through inspection of the correlation matrix of all items. For items on the same subscale correlations on the same sub-scale are recommended to be between .30 and .70 which was demonstrated.

Factor analysis, or factor extraction supported the inclusion of all eight items on the scale and two items emerged that explained $76 \%$ of the variance. A rotated factor matrix was used to further examine the underlying dimensionality of the factors. Based on the analysis, Factor I, Plans and Progress would include five items on the sub-scale: Daily Collaboration, Sufficient Resources, Team Support, Team Communication, and Strength and Skill of Team Members. Factor II, Team Communication would include All
Team Communication, Quality Communication and Plans and Progress.

Although a few comments were provided by the participants, they were directed to communication using technology. The CCRI did not incorporate survey items that address electronic communication among team members. Shirren and Philips [30] studied the use of technology to support interpersonal interaction. Thirty-nine employees completed a five-day communication diary recording their actual behavior when receiving personal and work-related e mails. The authors found that higher numbers of work-related e mails were related to higher levels of stress. Higher levels of negative affect were correlated to a delay in dealing with personal and work e mails.

Suggestions were made by the authors to provide systems that sorted and prioritized e mails and to use e mail more efficiently. The authors also recommended low quality e-mails be reduced and educating employees on responding to e mails in an adaptive manner was also suggested. Shirren and Philips [30] found that both technology and information are essential, however too much can be detrimental to productivity. Further development of the CCRI will incorporate scale items to rank individuals perceptions of communication and collaboration efforts through the use of technology.

Additional validation studies are required, including content review by a panel of experts. Further efforts to establish criterion validity are recommended, including efforts to correlate the new instrument with established scales. The value of well-constructed instruments cannot be understated.

\section{Summary}

The importance of identifying areas of concern among staff members caring for individuals receiving hospice care is critical. Collaboration and communication regarding end-of-life issues are key elements in assisting palliative patients and families an opportunity to redefine needs [13]. End-of-life communication requires support from the organization or institution. Experienced clinical providers, engaging in multi-professional teamwork and modeling effective communication skills are vital to improving end of life outcomes for the patient and family [17]. Blinderman and Prager [15] suggested that health care members can be taught active listening and can refine their communication skills to optimize patient preferences. Others have found that teamwork requires a detailed communication strategy that establishes effective sharing and flow of information. Demiris et al. [21] determined that improving personal communication requires an analysis of hospice team members to identify possible barriers to flow of information. Moving forward with efforts toward improving communication, collaboration and the avoidance of miscommunication begins with an accurate assessment of team members.

The CCRI provided a preliminary attempt to obtain measurement data regarding hospice team member's perceptions of the communication interaction on the admission team. Further development of the tool offers the potential to 
develop a reliable and valid instrument to measure perceptions of communication and collaboration. An instrument that is able to measure the areas where the clinical and non-clinical providers agree or

disagree on key elements of communication and collaboration is a necessary first step in assessing the strengths and weaknesses of the staff. Providers of health care are in key positions to assist the patient and their families deal with the impending death of a family member. Identification of problematic areas is vital to directing focus to areas that can benefit from additional education, institutional support and strategies to improve communication/ collaboration. Efforts to assess communication difficulties and resolve them will improve patient care and family concerns at a critical life transition.

\section{References}

1. King IM. Toward a theory for nursing: general concepts of human behavior. John Wiley \& Sons Canada, Limited; 1971.

2. King IM. A Theory for Nursing: Systems, Concepts, Process. Delmar; 1981.

3. http://www.nhpco.org/about/hospice-care

4. King IM, Fawcett J. The Language of Nursing Theory and Metatheory. Sigma Theta Tau International, Center Nursing Press; 1997.

5. Khowaja K. Utilization of King's interacting systems framework and theory of goal attainment with new multidisciplinary model: clinical pathway. Aust J Adv Nurs. 2006;24:17285836.

6. Kuebler KK, Bruera E. Interactive collaborative consultation model in end-of-life care. J Pain Symptom Manage. 2000;20:11018338.

7. Bronstein LR. A model for interdisciplinary collaboration. Soc Work. 2003;48:12899277.

8. Demiris G, Oliver DP, Wittenberg-Lyles E. Technologies to Support End of Life Care. Semin Oncol Nurs. 2011;27:21783012. doi:10.1016/j. soncn.2011.04.006

9. Bushinski RL, Cummings KM. Practices of effective end-of-life communication between nurses and patients/families in two care settings. Creat Nurs. 2007;13:18286973.

10. Corcoran AM, Casarett DJ. Improving communication and rethinking hospice care. Chest. 2010;137:20525649. doi:10.1378/chest.10-0040.

11. Dunne K. Effective communication in palliative care. Nurs Stand. 2005;20:16370506. doi:10.7748/ns2005.12.20.13.57.c4024.

12. Arnold BL. Mapping hospice patients' perception and verbal communication of end-of-life needs: an exploratory mixed methods inquiry. BMC Palliative Care. 2011;10. doi:10.1186/1472-684X-10-1.

13. Dale C. Communication in palliative care. Nurs Stand. 2006;20:16881590.

14. Blinderman CD, Prager K. The right to information--a first step toward improving end-of-life care. J Pain Symptom Manage. 2011;41:21444185. doi:10.1016/j.jpainsymman.2011.02.005.

15. Betcher DK. Elephant in the room project: improving caring efficacy through effective and compassionate communication with palliative care patients. Medsurg Nurs. 2010;19:20476520.

16. Clarke A, Ross H. Influences on nurses' communications with older people at the end of life: perceptions and experiences of nurses working in palliative care and general medicine. Int J Older People
Nurs. 2006;1:20925726. doi:10.1111/j.1748-3743.2006.00006.x.

17. Ashurst A. Palliative care: Effective communication. Nursing and Residential Care. 2007;9. doi:10.12968/nrec.2007.9.2.22746.

18. Wittenberg-Lyles EM, Oliver DP. The power of interdisciplinary collaboration in hospice. Progress in Palliative Care. 2007;15. doi:10.1179/096992607X177764.

19. Demiris G, Washington K, Oliver DP, Wittenberg-Lyles E. A study of information flowin hospice interdisciplinary team meetings. J Interprof Care. 2008;22:19012142. doi:10.1080/13561820802380027.

20. Wittenberg-Lyles EM, Gee GC, Oliver DP, Demiris G. What Patients and Families Don't Hear: Backstage Communication in Hospice Interdisciplinary Team Meetings. J Hous Elderly. 2009;23:20046944. doi:10.1080/02763890802665007.

21. Ponte PR, Gross AH, Milliman-Richard YJ, Lacey K. Interdisciplinary teamwork and collaboration: an essential element of a positive practice environment. Annu Rev Nurs Res. 2010;28:21639027.

22. Austin W, Park C, Goble E. From interdisciplinary to transdisciplinary research: a case study. Qual Health Res. 2008;18:18354053. doi:10.1177/1049732307308514.

23. McDonald C, McCallin A. Interprofessional collaboration in palliative nursing: what is the patient-family role? Int J Palliat Nurs. 2010;16:20925291. doi:10.12968/ijpn.2010.16.6.48832.

24. Kay S, Husbands E, Antrobus JH, Munday D. Provision for advanced pain management techniques in adult palliative care: a national survey of anaesthetic pain specialists. Palliat Med. 2007;21:17656403. doi:10.1177/0269216307078306.

25. Weinberg DB, Cooney-Miner D, Perloff JN, Babington L, Avgar AC. Building collaborative capacity: promoting interdisciplinary teamwork in the absence of formal teams. Med Care. 2011;49:21478768. doi:10.1097/MLR.0b013e318215da3f.

26. Green SD, Thomas JD. Interdisciplinary collaboration and the electronic medical record. Pediatr Nurs. 2008;34:18649812.

27. Dy SM, Roy J, Ott GE, McHale M, Kennedy C, Kutner JS, et al. Tell UsTM: a Web-based tool for improving communication among patients, families, and providersin hospice and palliative care through systematic data specification, collection, and use. J Pain Symptom Manage. 2011;42:21458214. doi:10.1016/j.jpainsymman.2010.12.006.

28. Shirren S, Phillips JG. Decisional style, mood and work communication: email diaries. Ergonomics. 2011;54:21973001. doi:10.1080/0014013 9.2011.609283.

29. Dawson S. Interprofessional working: communication, collaboration... perspiration! Int J Palliat Nurs. 2007;13:18073710. doi:10.12968/ ijpn.2007.13.10.27494.

30. Thannhauser J, Russell-Mayhew S, Scott C. Measures of interprofessional education and collaboration. J Interprof Care. 2010;24:20540613. doi:10.3109/13561820903442903.

31. Jünger S, Pestinger M, Elsner F, Krumm N, Radbruch L. Criteria for successful multiprofessional cooperation in palliative care teams. Palliat Med. 2007;21:17656412. doi:10.1177/0269216307078505.

32. McCaffrey R, Hayes RM, Cassell A, Miller-Reyes S, Donaldson A, Ferrell $C$. The effect of an educational programme on attitudes of nurses and medical residents towards the benefits of positive communication and collaboration. J Adv Nurs. 2012;68:21679224. doi:10.1111/j.13652648.2011.05736.x.

33. Plichta SB, Garzon LS. Statistics for Nursing and Allied Health. Lippincott Williams \& Wilkins; 2009. 